\title{
Research on Data Preprocessing and Categorization Technique for Smartphone Review Analysis
}

\author{
Vivek Agarwal \\ Department Of Computer Engineering, \\ Smt. Kashibai Navale \\ College of Engineering. \\ 'Ram Darshan' bldg. 46/D \\ Aundh Road,Pune -20
}

\begin{abstract}
Given the current market scenario, competition for dominance is at its peak. The best way for any smartphone manufacturer to deliver a quality product, is to perform a review analysis based on the end user experience and their demands. This helps the manufacturers to make necessary product changes and launch more relevant features in their smartphones. This paper aims to highlight the data preprocessing steps required for review analysis of a newly launched smartphones in the market by collecting tweets from the Twitter data feed. It aims to improvise the preprocessing steps and increase accuracy by filtering out the less relevant tweets and consider the more appropriate and genuine tweets.
\end{abstract}

\section{General Terms}

Categorization, reviews, iPhone, twitter, preprocessing, text.

\section{Keywords}

analysis, categorization, cleaning, dataframe, gibberish, preprocessing, processed, raw, review, structured, transformation.

\section{INTRODUCTION}

Today's market scenario is a lot different than what it used to be a decade ago. With the introduction of smartphones and inbuilt sensors, the competition for high profile processors, high resolution cameras, screen size and performance has sky rocketed. Against this backdrop, the manufacturers risk a lot when planning to launch a new device in the market. It is important to carry out a detailed survey regarding the response of the end users to the previously launched products, its shortcomings and suggested improvisation by experts. After incorporating such changes and feature improvements, the situation is a lot safer for manufacturers to launch the new device and probability of success increases exponentially. Twitter is one of the most commonly and widely used platforms for users who wish to express themselves, and there is often a sentiment associated with a user's tweet that expresses their likes and dislikes regarding their device experience. In this paper, I aim to target these tweets, categorize them according to various features that it expresses and apply preprocessing technique. The objective of this paper, is to demonstrate how Bot generated gibberish tweets can be separated from genuine human tweets and different aspects of a smartphone, can be categorized into the respective features that it talks about. For instance, a tweet saying, "Soo excited about my new iPhone 6S! Great camera and awesome processing speed! $\square$ \#iPhone6S \#Selfies \#Happy" expresses reviews regarding camera quality and processor performance. We need to categorize this tweet and apply preprocessing to transform this data into useful information. The data (tweets) that I have obtained was in an unstructured form. I have transformed this data by implementing a proper structure using dataframe, which allows us to apply SQL like queries to extract information.

\section{DATA PREPROCESSING}

Data Preprocessing is the process of simply transforming raw data into understandable format. Real world data is sometimes incomplete, inconsistent, redundant and noisy. Data preprocessing involves various steps that help to convert raw data into processed and sensible format.

The diagram below is used to depict the various steps involved in data preprocessing.

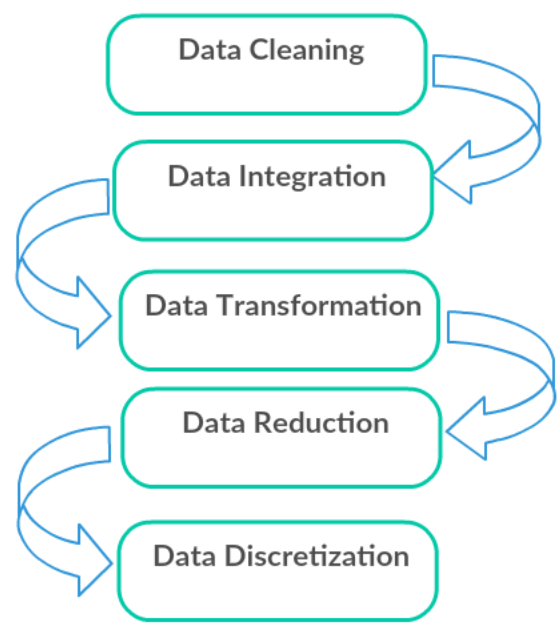

Fig 1. Data Preprocessing Steps.

\section{A. Data Cleaning}

Data cleaning is the process of detecting corrupt data and inaccurate records from a record set or database table. The main use of cleaning step is based on detecting incomplete, inaccurate, inconsistent and irrelevant data and applying techniques to modify or delete this useless data.

\section{B. Data Integration}

Data Integration focuses on unification of data residing in different sources and presenting a unified view of these data. Data with different representations are put together and any conflicts resulting from it are resolved. This process becomes vital in a number of scientific and commercial applications. With increasing volume and exponential growth of data, integrating it becomes even more significant.

\section{Data Transformation}

Data transformation plays a pivotal role in converting unprocessed data into understandable form. It consists of data normalization, aggregation and generalization. Data 
normalization helps to arrange the columns and tables of a database such that redundancy is minimum. This helps cut down on the processing time and complexity. Data aggregation helps in creating a brief summary for faster overview. The process of data generalization is also known as rolling-up data. It helps in generalizing data and creates successive layers of summary in evaluation database.

\section{Data Reduction}

Data reduction is the process of transforming digital info into ordered and simplified form. This data is generally derived through empirical and experimental means. It involves reducing large amounts of data into smaller and meaningful fragments.

\section{E. Data Discretization}

Data discretization is an important concept when you have a large amount of numeric data, but only want to classify it based on nominal values. In this scenario, the continuous data is split into discrete forms and the values of these discrete sets are said to be the nominal value. It is basically a process of converting continuous data attributes into a finite set of intervals with minimal loss of information.

\section{PROPOSED APPROACH}

In my approach, I have preprocessed the tweets that I received from twitter using tweepy API. The tweets that we receive are in unstructured JSON format. Our objective is to structure these tweets and preprocess it. I have mentioned the various steps involved in this process.

\subsection{Overview}

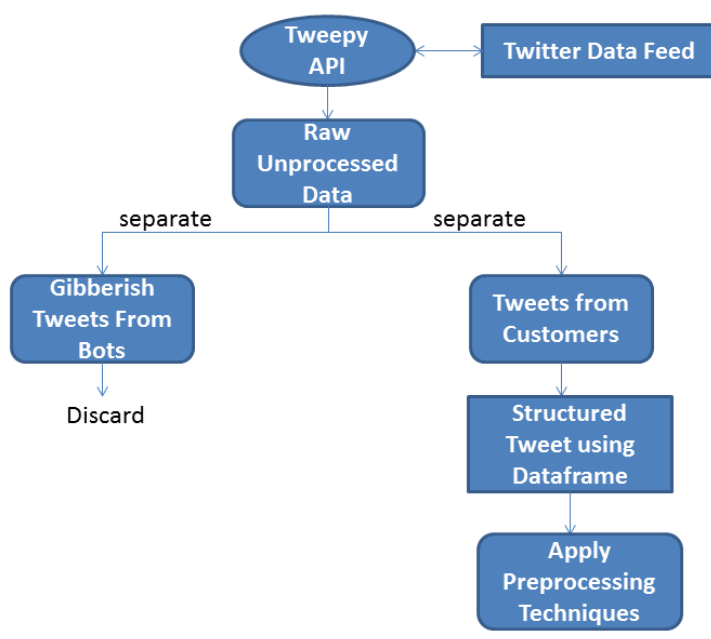

Fig 2. Overview of the entire procedure.

In my approach, I have used twitter data feed as my main source of dataset. Tweets obtained from twitter are extracted using the Tweepy API. This API is provided officially by twitter and is a RESTful API that requires special access token secret and consumer secret for authentication to access the live tweets. The tweets that we receive are obtained in a JSON format and have multiple attributes.

In order to search tweets relating to a particular device such as iPhone, I have set the filter as 'iPhone'. The Standard Listener then listens to all the tweets with 'iPhone' as the main keyword and hashtag. An example of sample JSON tweet is given as follows.

\{"created_at":"Sat Oct 24 05:48:17 +0000 2015", "id":657795727796531200, "id_str":"657795727796531200",
"text":"Love the HD resolution Camera for iPhone 6S. The body still looks a lil fragile to handle!", "source":"\u003ca href=l"http:Wtwitter.coml" rel=|"nofollowl"lu003eTwitter Web Clientlu003cValu003e", "truncated":false, "in_reply_to_status_id":null, "in_reply_to_status_id_str":null, "in_reply_to_user_id":null, "in_reply_to_user_id_str":null, "in_reply_to_screen_name":null, "user": \{ "id":2644475012, "id_str":"2644475012", "name":"richard", "screen_name":"farmview_SZ", "location":"\u53f0lu7063", "url":null, "description":"RV \& Trailer rear view system professional manufacture", "protected":false, "verified":false, "followers_count":2,

"friends_count": 18 ,"listed_count":0,"favourites_count":0,"stat uses_count":4, "created_at":"Mon Jul 14 08:20:07 +0000 2014","utc_offset":null,"time_zone":null,"geo_enabled":false, "lang":"zh-cn",

"favorite_count":0,"entities":\{ "hashtags":[],"urls":[\{"url":"htt ps:Wt.coVyW7Eq2ZUCI","expanded_url":"http:Wwww.ama zon.comVdpVB015GWJK8YVref=cm_sw_r_tw_dp_nzXkwb1 982M94","display_url":"amazon.comVdpVB015GWJK8Yhu 2026","indices":[81,104]\}],"user_mentions":[\{"screen_name" :"amazon","name":"Amazon","id":20793816,"id_str":"207938 16","indices":[108,115]\}],"symbols":[]\},"favorited":false,"ret weeted":false,"possibly_sensitive":false,"filter_level":"low","1 ang":"en","timestamp_ms":"1445665697417" \}

The "text" attribute used in this file contains the actual tweet that we are interested in and every other attribute is the metadata that we receive. In order to increase accuracy and decrease processing time, we can reduce the dataset by neglecting out the gibberish tweets. Many of the tweets that we obtain are generated by Bots which do not express any sentiment or review. These tweets are gibberish and not generated by humans. They need to be filtered out in order to reduce the size of the data. In order to identify the Bots, we make use of the "user" attribute of the JSON tweet. The Bots can be easily identified from the "name" attribute which consists of random alphanumeric characters as opposed to proper alphabetical strings. Also the source of the tweet gives us a good indication of the genuinity of the tweet. For example, a Bot tweet with a source attribute is given as follows -

"source":"lu003ca href=l"http:Wtwittbot.netV".

Here, we can trace the origin of the tweet to a twitter bot URL indicating a non-human generated tweet. In the previous example of JSON tweet, note that the "source" attribute contains "http:Wtwitter.coml" as the URL and "name" attribute is set to "richard" indicating a plausible human name.

\subsection{Structured Form.}

In order to define a structure for the tweets that we receive, I have defined it in a dataframe. To create a dataframe, I've made use of 'Pandas Data Analysis Library' in Python. Pandas is an open source, BSD-licensed library providing high performance, easy to use data structures and data analysis tool.

It can be used for reading and writing data between inmemory data structures and different formats: CSV and text files, Microsoft Excel and SQL databases.

Pandas Dataframe helps us structure all the tweets in various categories such as tweets relating to 'camera', 'screen', 'battery', 'app', 'processor', 'bend', 'connectivity' and 'performance' as the various categories. These categories also form the columns of our structured database table. Once we 
have categorized the tweets into their respective matching columns, we shall apply preprocessing techniques to each one.

This method of categorization is performed using synonym builder and bag of words clustering. Pandas also supports querying language for information extraction from dataframe. We can extract particular columns and/or rows using querying.

\subsection{Preprocessing Technique}

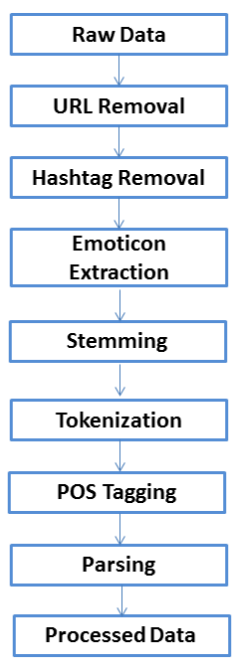

Fig 3. Preprocessing Steps

The data that we have obtained from twitter data feed is in a raw unprocessed JSON format. Our objective would be to process this data in a cleaner format such that we can extract information further. Fig 3 demonstrates the step by step process of the techniques that we plan to apply to the data for preprocessing.

The tweepy API is a restful web service that extracts live tweets from twitter database. This collection of tweets is saved into a JSON file in our computer that acts as our primary source of dataset. In order to achieve high precision and accuracy, I have saved a large amount of dataset.

In order to increase accuracy of the reviews that we plan to extract at a later stage, we need to filter this dataset. The tweets that we have obtained contain gibberish tweets that have been created by BOTs rather than humans, as demonstrated in Fig 2. It is vital to filter out these kinds of tweets as they can cause noise and irrelevancies in our results. Filtering out these tweets can help us get a closer estimation of human reviews.

\subsubsection{URL Removal}

The urls used in the tweet play no vital role in conveying the sentiment behind a user's review. It thus needs to be discarded. We make use of regular expression to find the occurrence of a hyperlink. Regular expressions library helps in pattern matching and url detection. Once a link has been detected, it is discarded from the tweet.

\subsubsection{Hashtag Removal}

The hashtags used in the tweets denote the subject that the tweet is about. In this analysis I have removed neutral hashtags and tokenized the ones demonstrating sentiments
Table I. Examples showing the tweets and their corresponding expression denoted using hashtags.

\begin{tabular}{|c|c|}
\hline Tweet & Expression \\
\hline $\begin{array}{c}\text { "Got a new iPhone 6S } \\
\text { \#awesome \#happyyy" } ;\end{array}$ & $\begin{array}{c}\text { Hashtags represent 2 } \\
\text { positive and 1 neutral } \\
\text { expressions. }\end{array}$ \\
\hline $\begin{array}{c}\text { "The back cover could } \\
\text { be a lil darker! } \\
\text { \#iPhone6S \#nevermind" }\end{array}$ & $\begin{array}{c}\text { Hashtags represents 2 } \\
\text { neutral expressions } \\
\text { relating to back cover. }\end{array}$ \\
\hline $\begin{array}{c}\text { "RT @jason123: thanks } \\
\text { for the new iPhone bday } \\
\text { gift!! Yay! \#iPhone } \\
\text { \#Happpyy" }\end{array}$ & $\begin{array}{c}\text { Hashtags represents 1 } \\
\text { neutral and 1 positive } \\
\text { expression. }\end{array}$ \\
\hline
\end{tabular}

\subsubsection{Emotion extraction}

The emoticons play a vital role in conveying the sentiment of the end-user regarding his product experience. For example, ":-)" is labeled as positive whereas ":(" is labeled as negative. We assign each emoticon a label as follows: Extremelypositive, Extremely-negative, Positive, Negative, and Neutral.

\subsubsection{Stemming}

Stemming is the process of reducing the derived words to the word's stem. For example, words like thoughtful, doggy, catlike, exciting are reduced to thought, dog, cat, excite as their stem words. In order to match the synonyms of the stems, I have used a synonym builder. For example, synonyms of 'excite' are 'thrilled', 'exhilarated', 'animated', 'electrified', 'moved' etc. Stemming helps reduce a large variety of words into a single word denoting its stem or synonym.

In this, I have also expanded the commonly used acronyms into their English equivalent words.

Table 2. Examples denoting the acronym and corresponding expansion.

\begin{tabular}{|c|c|}
\hline Acronym & English Expansion \\
\hline gr8, gr8t & Great \\
\hline lol & Laughing out loud \\
\hline rofl & $\begin{array}{c}\text { Rolling on the floor } \\
\text { laughing. }\end{array}$ \\
\hline
\end{tabular}

I have also replaced sequence of repeated characters by three characters. For example, we convert 'awsoooome' to 'awesooome' and 'coooool' to 'coool'. We do not replace it by 2 characters as we wish to differentiate between regular usage and emphasized usage. I have made use of regular expressions library in python for reduction.

\subsubsection{Tokenization}

Tokenization is the process of creating tokens for the words obtained in the tweet. The entire sentence is split into fragmented words which can later be tagged for POS analysis and parse tree generation. For example, consider the tweet -

"RT@SkyNews: Facebook admits its app drains iPhone battery but says a fix is here https:Wt.coVoHDFrSwaGa https:Wt.coVbnWVUIVjy0"

The tokenized form that I obtained is as follows 
[u'Facebook', u'admits', u'its', u'app', u'drains', u'iPhone', u'battery', u'but', u'says', u'a', u'fix', u'is', u'here']

This shows that the tweet has been successfully rid of 'RT@ SkyNews:' and the URL links. I have made use of Natural Language Toolkit library to perform tokenization.

\subsubsection{POS Tagging}

POS (Parts of Speech) Tagging, also known as word-category disambiguation, is the technique of marking up a word in corpus as corresponding to a part of speech. Using NLTK library, I have created the POS tags for the tokens earlier generated.

For the tweet mentioned in the previous step, here are the resultant POS tagged words.

[(u'Facebook', 'NNP'), (u'admits', 'VBZ'), (u'its', 'PRP\$'), (u'app', 'NN'), (u'drains', 'VBZ'), (u'iPhone', 'JJ'), (u'battery', 'NN'), (u'but', 'CC'), (u'says', 'VBZ'), (u'a', 'DT'), (u'fix', 'NN'), (u'is', 'VBZ'), (u'here', 'RB')]

\subsubsection{Parsing}

After we are done generating token and tagging them, our final step would be to perform Semantic analysis. For this we need to define a CFG and generate a parse tree accordingly.

For this purpose I have used the Penn Treebank corpus. If the token is a stop-word, we simply add a subtree "(STOP ('stopword'))" to the parse tree. These subtrees need not be traversed when looking for the review sentiment. Before constructing parse trees I have generated named entities for the processed tweets. One example of named entities for the above tweet is
(S (ORGANIZATION Facebook/NNP) admits/VBZ its/PRP\$ app/NN drains/VBZ (ORGANIZATION iPhone/JJ) battery/NN but/CC says/VBZ a/DT fix/NN is/VBZ here/RB)

Notice that the entities Facebook and iPhone are named as ORGANIZATION.

\section{RESULTS}

The preprocessing has been applied on a variety of smartphones such as iPhone, OnePlus Two, Samsung Galaxy S6. Below are the results obtained after compiling the tweets about the above mentioned devices.

Table 3. Demonstrates the statistics involved in the analysis.

\begin{tabular}{|c|c|c|}
\hline $\begin{array}{c}\text { Total Tweets } \\
\text { Analyzed }\end{array}$ & $\begin{array}{c}\text { Tweets from BOTS } \\
\text { (Gibberish) }\end{array}$ & Meaningful tweets \\
\hline 56,436 & 13,248 & 43,188 \\
\hline
\end{tabular}

After the tweets have been extracted categorization is applied. The tweets are categorized according to the top features that they review.

Table 4. Total numbers of tweets in each category obtained.

\begin{tabular}{|c|c|c|c|}
\hline Applications & Camera & Screen & Battery \\
\hline 5,966 & 6,649 & 5,506 & 6,131 \\
\hline
\end{tabular}

Table 5. Total number of tweets in each category obtained.

\begin{tabular}{|c|c|c|c|}
\hline Performance & iOS & Android & $\begin{array}{c}\text { Processing } \\
\text { and Memory }\end{array}$ \\
\hline 4,125 & 6,489 & 3,965 & 4,369 \\
\hline
\end{tabular}

Table 6 . Top Tweets before Preprocessing

\begin{tabular}{|c|c|c|c|}
\hline Applications & Camera & Screen & Battery \\
\hline $\begin{array}{l}\text { "Do you guys } \\
\text { know how to make } \\
\text { app shortcuts on } \\
\text { iphone? Got some } \\
\text { trouble figuring it } \\
\text { out" }\end{array}$ & $\begin{array}{l}\text { OnePlus } 2 \text { Review: It's } \\
\text { Powerful, It's Cheap, and } \\
\text { great camera resolution!! } \\
\text { http://dlvr.it/BxynDl } \\
\text { \#oneplustwo \#oneplus } \\
\text { \#mobile \#oneplustworeview } \\
\text { \#lifehacker \#tech }\end{array}$ & \begin{tabular}{lr}
\multicolumn{1}{c}{ iPhone } & $6 \mathrm{~S}$ nano \\
review: & lightning \\
fast, & slightly \\
heavier, & 3D Touch \\
Screen is great.
\end{tabular} & $\begin{array}{l}\text { One plus two } \\
\text { battery life problems } \\
\text { are hard to deal with } \\
:(\ldots \text { \#onePlusTwo } \\
\text { \#battery }\end{array}$ \\
\hline $\begin{array}{l}\text { "Happy with the } \\
\text { \#OnePlusTwo, Sad } \\
\text { that it has Bugs.. } \\
\text { Hoping it's resolved } \\
\text { soon", }\end{array}$ & $\begin{array}{l}\text { I traded my Galaxy S5 for } \\
\text { a Galaxy Note } 5 . \text { Better } \\
\text { camera, faster processor, } \\
\text { faster charging. It only cost } 2 \\
\text { years! }\end{array}$ & $\begin{array}{l}\text { \#LG LG V10 vs } \\
\text { Samsung Galaxy } \\
\text { Note 5: LG's dual- } \\
\text { screen handset has } \\
\text { better selfie camera. }\end{array}$ & $\begin{array}{l}\text { Facebook } \\
\text { addresses severe } \\
\text { battery drain issue } \\
\text { with updated iOS app }\end{array}$ \\
\hline $\begin{array}{l}\text { "RT@SkyNews: } \\
\text { Facebook admits its } \\
\text { app drains iPhone } \\
\text { battery but says a fix } \\
\text { is here } \\
\text { https://t.co/oHDFrSw } \\
\text { aGa } \\
\text { https://t.co/bnWVUIV } \\
\text { jy0" }\end{array}$ & $\begin{array}{l}\text { \#LG LG V10 vs Samsung } \\
\text { Galaxy Note 5: LG's dual- } \\
\text { screen handset has better } \\
\text { selfie camera. }\end{array}$ & $\begin{array}{l}\text { sometimes the } \\
\text { screen of my } \\
\text { \#OnePlusTwo turns } \\
\text { black and I have to } \\
\text { restart the phone, } \\
\text { Any } \\
\text { recomendations? }\end{array}$ & $\begin{array}{l}\text { \#oneplustwo a } \\
\text { device that doesn't let } \\
\text { u down except for the } \\
\text { Battery, barely } 6: 30 \\
\text { hrs backup. Truly } \\
\text { Beast on } \\
\text { performance }\end{array}$ \\
\hline
\end{tabular}


Table 7 . Top Tweets before Preprocessing (Cont.)

\begin{tabular}{|c|c|c|c|}
\hline Performance & iOS & Android & $\begin{array}{l}\text { Processing and } \\
\text { Memory }\end{array}$ \\
\hline $\begin{array}{l}\text { The bend actually } \\
\text { scares me, I know } \\
\text { everybody's iPhone } 6 \text { does } \\
\text { bend eventually but it's so } \\
\text { worrying!! }\end{array}$ & $\begin{array}{l}\text { Looking forward } \\
\text { to the iOS update. } \\
\text { \#iOS \#iPhone }\end{array}$ & $\begin{array}{l}\text { The cynogen } \\
\text { replaced Oxygen OS } \\
\text { for android is quite } \\
\text { impressive. } \\
\text { \#OnePlusTwo } \\
\text { \#Oxygen }\end{array}$ & $\begin{array}{l}\text { The \#OnePlusTwo } \\
\text { finally arrived for my } \\
\text { dad. The packaging is } \\
\text { super sleek! Processor } \\
\text { seems fast. This one is a } \\
\text { \#winner }\end{array}$ \\
\hline $\begin{array}{l}\text { Does anyone have any } \\
\text { thoughts on \#OnePlusTwo } \\
\text { Especially regarding the } \\
\text { heating performance } \\
\text { issues? }\end{array}$ & $\begin{array}{l}\text { is my phone } \\
\text { broken or is iOS } 9 \\
\text { possibly this } \\
\text { buggy? why??? }\end{array}$ & $\begin{array}{l}\text { One thing I like } \\
\text { about \#oneplustwo is it } \\
\text { handles notifications } \\
\text { beautifully! } \\
\text { @OnePlus_IN I've } \\
\text { used android phns be4 } \\
\text { but this phone is } \\
\text { unique }\end{array}$ & $\begin{array}{l}\text { LG V10 vs Samsung } \\
\text { Galaxy S6 Edge Plus: } \\
\text { Samsung's device has } \\
\text { faster processor }\end{array}$ \\
\hline $\begin{array}{l}\text { Happy with my one } \\
\text { plus two @OnePlus_IN } \\
\text { butter } \\
\text { performance }\end{array}$ & $\begin{array}{l}\text { The "Moments" } \\
\text { tab in the Twitter } \\
\text { iOS update is trash. } \\
\text { \#iPhone } \\
\text { \#iOS }\end{array}$ & $\begin{array}{l}\text { @iamkaran24 } \\
\text { @dumbb786 The best } \\
\text { Mobile for Dual Sim } \\
\text { android I recommend } \\
\text { Is One Plus Two. }\end{array}$ & $\begin{array}{l}\text { Galaxy Note } 5 \\
\text { Stunning } \\
\text { design, gorgeous display, } \\
\text { power packed processor, } \\
\text { Multitasking and stylus. }\end{array}$ \\
\hline
\end{tabular}

Table 8. Top Tweets after Preprocessing

\begin{tabular}{|c|c|c|c|}
\hline Applications & Camera & Screen & Battery \\
\hline $\begin{array}{l}\text { [('Do', 'VB'), ('you', } \\
\text { 'PRP'), ('guys', 'VB'), } \\
\text { ('know', 'VB'), ('how', } \\
\text { 'WRB'), ('to', 'TO'), } \\
\text { ('make', 'VB'), ('app', } \\
\text { 'JJ'), ('shortcuts', } \\
\text { 'NNS'), ('on', 'IN'), } \\
\text { ('iphone', 'NN'), ('Got', } \\
\text { 'NNP'), ('some', 'DT'), } \\
\text { ('trouble', 'NN'), } \\
\text { ('figuring', 'VBG'), ('it', } \\
\text { 'PRP'), ('out', 'RP')] }\end{array}$ & $\begin{array}{l}\text { [('OnePlus', 'CC'), } \\
\text { ('2', 'CD'), ('Review', } \\
\text { 'NN'), ('Its', 'PRP'), } \\
\text { ('Powerful', 'NNP'), } \\
\text { ('It', 'PRP'), ('"s", } \\
\text { 'VBZ'), ('Cheap', } \\
\text { 'NNP'), ('and', 'CC'), } \\
\text { ('great', 'JJ'), ('camera', } \\
\text { 'NN'), ('resolution', } \\
\text { 'NN'), ] }\end{array}$ & $\begin{array}{l}\text { [('iPhone', 'NN'), ('6S', } \\
\text { 'CD'), ('nano', 'JJ'), } \\
\text { ('review', 'NN'), } \\
\text { ('lightning', 'NN'), ('fast', } \\
\text { 'RB'), ('slightly', 'RB'), } \\
\text { ('heavier', 'JJR'), ('3D', } \\
\text { 'CD'), ('Touch', 'NNP'), } \\
\text { ('Screen', 'NNP'), ('is', } \\
\text { 'VBZ'), ('great', 'JJ')] }\end{array}$ & $\begin{array}{l}\text { [('One', 'CD'), ('plus', } \\
\text { 'CC'), ('two', 'CD'), } \\
\text { ('battery', 'NN'), ('life', } \\
\text { 'NN'), ('problems', } \\
\text { 'NNS'), ('are', 'VBP'), } \\
\text { ('hard', 'JJ'), ('to', 'TO'), } \\
\text { ('deal', 'VB'), ('with', } \\
\text { 'IN')] }\end{array}$ \\
\hline \begin{tabular}{l}
\multicolumn{1}{c}{ [('Happy', 'JJ'), } \\
('with', 'IN'), ('the', \\
'DT'),('Sad', 'NNP'), \\
('that', 'IN'), ('it', 'PRP'), \\
('has', 'VBZ'), ('Bugs..', \\
'VBN'), ('Hoping', \\
'VBG'), ('it', 'PRP'), \\
(''s", \\
('resolved', 'VBZ'), \\
('soon', 'RB')]
\end{tabular} & 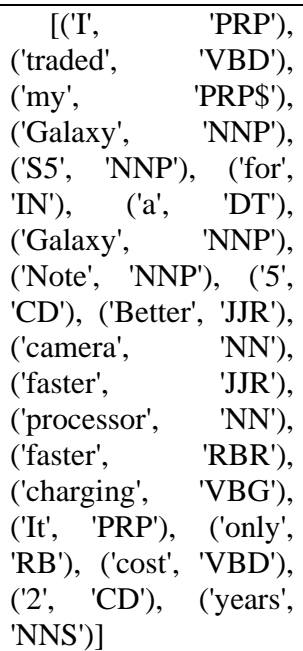 & $\begin{array}{l}\text { [('LG', 'NNP'), ('V10', } \\
\text { 'NNP'), ('vs', 'NN'), } \\
\text { ('Samsung', } \\
\text { ('Galaxy', 'NNP'), ('Note', } \\
\text { 'NNP'), ('5', 'CD'),('LG', } \\
\text { 'NNP'), ('dual-screen', } \\
\text { 'JJ'), ('handset', 'NN'), } \\
\text { ('has', 'VBZ'), ('better', } \\
\text { 'JJR'), ('selfie', 'VBN'), } \\
\text { ('camera', 'NN')] }\end{array}$ & $\begin{array}{l}\text { [('Facebook', 'NNP'), } \\
\text { ('addresses', 'VBZ'), } \\
\text { ('severe', 'JJ'), ('battery', } \\
\text { 'NN'), ('drain', 'NN'), } \\
\text { ('issue', 'NN'), ('with', } \\
\text { 'IN'), ('updated', 'JJ'), } \\
\text { ('iOS', 'NN'), ('app', } \\
\text { 'NN')] }\end{array}$ \\
\hline
\end{tabular}




\begin{tabular}{|c|c|c|c|}
\hline $\begin{array}{l}\text { [('Facebook', 'NNP'), } \\
\text { ('admits', 'VBZ'), ('its', } \\
\text { 'PRP\$'), ('app', 'NN'), } \\
\text { ('drains', 'VBZ'), } \\
\text { ('iPhone', 'JJ'), ('battery', } \\
\text { 'NN'), ('but', 'CC'), } \\
\text { ('says', 'VBZ'), ('a', } \\
\text { 'DT'), ('fix', 'NN'), ('is', } \\
\text { 'VBZ'), ('here', 'RB')] }\end{array}$ & \begin{tabular}{lr}
\multicolumn{1}{c}{ ('LG', 'NNP'), } \\
('V10', 'NNP'), ('vs', \\
'NN'), ('Samsung', \\
'NNP'), ('Galaxy', \\
'NNP'), ('Note', \\
'NNP'), ('5', 'CD'), \\
('LG', 'NNP'), ('dual- \\
screen', r 'JJ'), \\
('handset', 'NN'), \\
('has', 'VBZ'), ('better', \\
'JJR'), ('selfie', 'VBN'), \\
('camera', 'NN')]
\end{tabular} & $\begin{array}{l}\text { [('sometimes', 'RB'), } \\
\text { ('the', 'DT'), ('screen', } \\
\text { 'NN'), ('of', 'IN'), ('my', } \\
\text { 'PRP\$'), ('OnePlusTwo', } \\
\text { 'NNP'), ('turns', 'VBZ'), } \\
\text { ('black', 'JJ'), ('and', 'CC'), } \\
\text { ('I', 'PRP'), ('have', 'VBP'), } \\
\text { ('to', 'TO'), ('restart', 'VB'), } \\
\text { ('the', 'DT'), ('phone', } \\
\text { 'NN'), ('Any', 'DT'), } \\
\text { ('recomendations', 'NNS')] }\end{array}$ & $\begin{array}{l}\text { [('a', 'DT'), ('device', } \\
\text { 'NN'), ('that', 'WDT'), } \\
\text { ('does', 'VBZ'), ("n't", } \\
\text { 'RB'), ('let', 'VB'), ('u', } \\
\text { 'VB'), ('down', 'RP'), } \\
\text { ('except', 'IN'), ('for', } \\
\text { 'IN'), ('the', 'DT'), } \\
\text { ('Battery', } \\
\text { ('barely', 'RB'), ('6:30', } \\
\text { 'CD'), ('hrs', 'NN'), } \\
\text { ('backup', 'NN'), ('Truly', } \\
\text { 'NNP'), ('Beast', 'NNP'), } \\
\text { ('on', } \\
\text { ('performance', 'NN')] }\end{array}$ \\
\hline
\end{tabular}

Table 9. Top Tweets after Preprocessing

\begin{tabular}{|c|c|c|c|}
\hline Performance & iOS & Android & $\begin{array}{l}\text { Processing and } \\
\text { Memory }\end{array}$ \\
\hline $\begin{array}{l}\text { [('The', 'DT'), ('bend', } \\
\text { 'NN'), ('actually', 'RB'), } \\
\text { ('scares', 'VBZ'), ('me', } \\
\text { 'PRP'), ('I', 'PRP'), } \\
\text { ('know', 'VBP'), } \\
\text { ('everybody', 'NN'), ("'s", } \\
\text { 'POS'), ('iPhone', 'NN'), } \\
\text { ('6', 'CD'), ('does', 'VBZ'), } \\
\text { ('bend', 'VB'), } \\
\text { ('eventually', 'RB'), ('but', } \\
\text { 'CC'), ('it', 'PRP'), ("'s", } \\
\text { 'VBZ'), ('so', 'RB'), } \\
\text { ('worrying', 'JJ')] }\end{array}$ & $\begin{array}{l}\text { [('Looking', } \\
\text { 'VBG'), ('forward', } \\
\text { 'RB'), ('to', 'TO'), } \\
\text { ('the', 'DT'), ('iOS', } \\
\text { 'NN'), ('update', } \\
\text { 'NN')] }\end{array}$ & $\begin{array}{l}\text { [('The', } \\
\text { ('cynogen', } \\
\text { ('replaced', 'NN'), } \\
\text { ('Oxygen', 'NNP'), ('OS', } \\
\text { 'NNP'), ('for', 'IN'), } \\
\text { ('android', 'NN'), ('is', } \\
\text { 'VBZ'), ('quite', 'RB'), } \\
\text { ('impressive', 'JJ')] }\end{array}$ & $\begin{array}{l}\text { [('The', 'DT'), ('finally', } \\
\text { 'RB'), ('arrived', 'VBD'), } \\
\text { ('for', 'IN'), ('my', 'PRP\$'), } \\
\text { ('dad', 'NN'), ('The', 'DT'), } \\
\text { ('packaging', 'NN'), ('is', } \\
\text { 'VBZ'), ('super', 'JJ'), } \\
\text { ('sleek', 'JJ'), ('Processor', } \\
\text { 'NNP'), ('seems', 'VBZ'), } \\
\text { ('fast', 'RB'), ('This', 'DT'), } \\
\text { ('one', 'NN'), ('is', 'VBZ'), } \\
\text { ('a', 'DT'), ('winner', 'NN')] }\end{array}$ \\
\hline $\begin{array}{l}\text { [('Does', } \\
\text { ('anyone', 'NN'), ('have', } \\
\text { 'VB'), ('any', } \\
\text { ('thoughts', 'NNS'), ('on', } \\
\text { 'IN'), ('Especially', 'RB'), } \\
\text { ('regarding', 'VBG'), ('the', } \\
\text { 'DT'), ('heating', 'NN'), } \\
\text { ('performance', 'NN'), } \\
\text { ('issues', 'NNS')] }\end{array}$ & $\begin{array}{l}\text { [('is', 'VBZ'), } \\
\text { ('my', 'PRP\$'), } \\
\text { ('phone', 'NN'), } \\
\text { ('broken', 'NN'), ('or', } \\
\text { 'CC'), ('is', 'VBZ'), } \\
\text { ('iOS', 'JJ'), ('9', } \\
\text { 'CD'), ('possibly', } \\
\text { 'RB'), ('this', 'DT'), } \\
\text { ('buggy', 'NN'), } \\
\text { ('why', 'WRB')] }\end{array}$ & $\begin{array}{l}\text { [('One', 'CD'), ('thing', } \\
\text { 'NN'), ('I', 'PRP'), ('like', } \\
\text { 'VBP'), ('about', 'RB'), } \\
\text { ('is', 'VBZ'), ('it', 'PRP'), } \\
\text { ('handles', 'VBZ'), } \\
\text { ('notifications', 'NNS'), } \\
\text { ('beautifully', 'RB'), ('I', } \\
\text { 'PRP'), ("'ve", 'VBP'), } \\
\text { ('used', 'VBN'), ('android', } \\
\text { 'JJ'), ('phns', 'NN'), ('be4', } \\
\text { 'NN'), ('but', 'CC'), ('this', } \\
\text { 'DT'), ('phone', 'NN'), ('is', } \\
\text { 'VBZ'), ('unique', 'JJ')] }\end{array}$ & 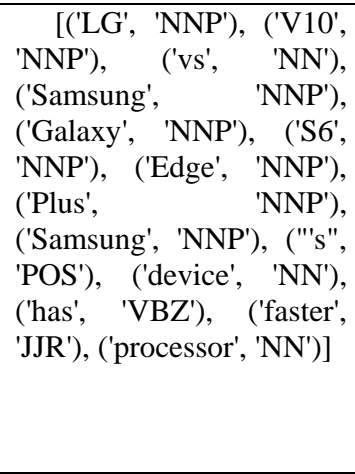 \\
\hline $\begin{array}{l}\text { [('Happy', 'JJ'), ('with', } \\
\text { 'IN'), ('my', 'PRP\$'), } \\
\text { ('one', 'CD'), ('plus', 'CC'), } \\
\text { ('two', 'CD'), ('butter', } \\
\text { 'NN'), ('smooth', 'VBD'), } \\
\text { ('performance', 'NN')] }\end{array}$ & $\begin{array}{l}\text { [('The', 'DT'), } \\
\text { ('Moments', } \\
\text { 'NNPS'), ('tab', } \\
\text { 'NN'), ('in', 'IN'), } \\
\text { ('the', } \\
\text { ('Twitter', 'NNP'), } \\
\text { ('iOS', 'NN'), } \\
\text { ('update', 'NN'), ('is', } \\
\text { 'VBZ'), ('trash', 'JJ')] }\end{array}$ & $\begin{array}{l}\text { [('The', 'DT'), ('best', } \\
\text { 'JJS'), ('Mobile', 'NN'), } \\
\text { ('for', 'IN'), ('Dual', } \\
\text { 'NNP'), ('Sim', 'NNP'), } \\
\text { ('android', 'NN'), ('I', } \\
\text { 'PRP'), ('recommend', } \\
\text { 'VBP'), ('Is', 'VBZ'), } \\
\text { ('One', 'CD'), ('Plus', } \\
\text { 'CC'), ('Two', 'CD')] }\end{array}$ & \begin{tabular}{lr}
\multicolumn{1}{c}{ [('Galaxy', } & 'NNP'), \\
('Note', 'NNP'), ('5', 'CD'), \\
('-Stunning', & 'VBG'), \\
('premium', & 'NN'), \\
('design', & 'NN'), \\
('gorgeous', & 'JJ'), \\
('display', 'NN'), ('power', \\
'NN'), ('packed', 'VBD'), \\
('processor', \\
('Multitasking', \\
('and', 'NN'), 'CC'), ('stylus', \\
'NN')]
\end{tabular} \\
\hline
\end{tabular}

\section{FUTURE SCOPE}

In this paper, I have taken into consideration, English as the primary source of language. However, NLTK supports multiple languages and thus, we can expand this work into a multi-lingual frame. The review accuracy can be increased by considering tweets from multiple languages such as German, French or Spanish.The algorithm used in this process does not take into consideration the concept of sarcastic tweets. For example, a tweet such as "The battery dries up in the blink of an eye. \#great" expresses sarcasm. However, this algorithm may fail to take it into consideration. We can further improvise on this system by modifying the algorithm to understand sarcasm.My next task would be based on using these categorized and processed tweets to classify and extract sentiment. 


\section{CONCLUSION}

In this paper, I have focused on tweet categorization and preprocessing. I have been able to successfully categorize the tweets depending upon the various features of the smartphones they are reviewing about.

This paper demonstrates that the dataset can be reduced by discarding the gibberish tweets from bots and focusing on the human tweets. This reduces processing complexity and gives a cleaner dataset.

The analysis has been carried out for a wide variety of smartphone manufacturers and a combined result has been demonstrated. Using the concept of categorized review analysis, the manufacturers can be given a segmented and detailed review of the various features pertaining to their device. This gives them more accurate analysis regarding the strengths and weaknesses of many features in particular.

\section{AKNOWLEDGEMENT}

I would like to whole heartedly thank, our professor, Mr. Pramod Mali, and our mentor Mr. Shital Patil in helping me find the right directions and important resources required to complete this project. A special thanks to Mrs. Aaradhna Deshmukh, for being my mentor and motivating me. I would like to thank my institiution and Head Of Computer Department, Mr. P.N. Mahalle for giving me access to the required resources and backing me all the while. Without the kindness of my institute and blessing of my professors, I would not have been able to complete this project.

\section{REFERENCES}

[1] Bird, Steven, Edward Loper and Ewan Klein (2009), Natural Language Processing with Python. O'Reilly Media Inc.

[2] Sumian Peng, "Research on Data Preprocessing Process in the Web Log Mining," IEEE Conference. Information Science and Engineering (ICISE), 2009 1st International Conference, pp. 942 - 945, 26-28 Dec. 2009

[3] Sun Binli,"Research on data-preprocessing for construction of university information systems", IEEE
Conference. Computer Application and System Modeling (ICCASM), 2010 International Conference Volume:1. 22-24 Oct. 2010

[4] Sudheer Reddy, K," An effective data preprocessing method for Web Usage Mining", IEEE Conference Information Communication and Embedded Systems (ICICES), 2013 International Conference. 7 - 10 Feb. 2013.

[5] Wei Jianping, "Research on Data Preprocessing in Supermarket Customers Data Mining", IEEE Conference Information Engineering and Computer Science (ICIECS), 2010 2nd International Conference, pp. 1 - 4, 25-26 Dec. 2010

[6] Qing Ang, "Explored research on data preprocessing and mining technology for clinical data applications", IEEE Conference. Information Management and Engineering (ICIME), 2010 The 2nd IEEE International Conference, pp 327 - 330.16-18 April 2010.

[7] García, Salvador, "Data Preprocessing in Data Mining", Intelligent Systems Reference Library.

[8] Wei Zhang, "Data Preprocessing for Web Data Mining", Advances in Electronic Commerce, Web Application and Communication, pp 303-307.

[9] Sharon Christa, "Data Preprocessing Using Intelligent Agents", Proceedings of the International Conference on Frontiers of Intelligent Computing: Theory and Applications (FICTA), pp 197-204.

[10] Gaston L'Huillier, "Web Usage Data Pre-processing", Advanced Techniques in Web Intelligence-2, pp 11-34

[11] Amir R. Razavi, "A Data Pre-processing Method to Increase Efficiency and Accuracy in Data Mining", 10th Conference on Artificial Intelligence in Medicine, pp 434-443. 